\title{
Performance of Convolution Coded Multicarrier CDMA System in Frequency Selective Rayleigh Channel
}

\author{
Anita Mukati \\ PG Scholar \\ Department of ECE \\ IES, IPS Academy, Indore,M.P.,India
}

\author{
Angeeta Hirwe \\ Associate Professor \\ Department of ECE \\ IES, IPS Academy, Indore,M.P.,India
}

\begin{abstract}
The paper describes the performance of convolution coded Multicarrier Code Division Multiple Access (MC-CDMA) system evaluated for different equalization techniques i.e. Maximal Ratio Combining (MRC) and Equal Gain Combining (EGC) in frequency selective Rayleigh channel in detail. For symbol mapping two modulation techniques: Binary Phase Shift Keying (BPSK) and Quadrature Phase Shift Keying (QPSK) is used. The comparison is done between the performances of MC-CDMA system with convolution codes and without convolution codes for different equalization and modulation techniques in terms of Bit Error Rate (BER) and Signal to Noise Ratio (SNR) using MATLAB based simulation. Simulation results reveal better SNRs when $1 / 2$ rate convolution coding is used with different modulation techniques.
\end{abstract}

\section{Keywords}

Convolution Code, MC-CDMA, MRC, EGC, BPSK, QPSK

\section{INTRODUCTION}

There is a growing need of technological innovations to fulfill the surging increase in the demand for high-rate-data transmission requirement of the next generation personal wireless radio communications [1]. Multicarrier Code Division Multiple Access (MC-CDMA) is a promising technique for next generation diverse mobile network which is also known as OFDM-CDMA because it combines the benefits of Code Division Multiple Access (CDMA) and Orthogonal Frequency Division Multiplexing (OFDM) system [2][3][4].

In this paper the performance of MC-CDMA system has compared for with and without convolution codes in frequency selective channel environment by considering different number of users with BPSK and QPSK modulation schemes and two combining techniques

The paper is organized as follows: In Section 2, the model of the transmission system used is given. Simulation parameter and simulation results are discussed In Section 3. A brief conclusion is given in Section 4.

\section{SYSTEM MODEL}

\subsection{MC-CDMA system}

Considered MC-CDMA system model is shown in Figure it has $\mathrm{N}$ users $(2,8$ and 16) with corresponding $\mathrm{N}$ sequences of symbols. Different sequences are obtained after spreading the convolutional coded and modulated data of each user.

At the transmitter section user's binary data is encoded using $1 / 2$ rate convolution encoder after encoding, the encoded data modulated by different modulation techniques (i.e. BPSK, QPSK). Spreading of the complex valued modulated sequence dk is done using Walsh- Hadamard codes with code length $\mathrm{L}=16$

$\mathrm{C}(\mathrm{k})=\{\mathrm{c} 0(\mathrm{k}), \mathrm{c} 1(\mathrm{k}), \mathrm{c} 2(\mathrm{k}), \ldots$ $. \mathrm{c} 15(\mathrm{k})\} \mathrm{T}$

Spreading of the sequence results a complex valued sequence in vector notations which is given by

$\mathrm{S}(\mathrm{k})=\mathrm{d}(\mathrm{k}) \mathrm{C}(\mathrm{k})=\{\mathrm{s} 0(\mathrm{k}), \mathrm{s} 1(\mathrm{k}), \mathrm{s} 2(\mathrm{k}), \ldots . \mathrm{s} 15(\mathrm{k})\} \mathrm{T} \ldots \ldots .(2)$

The MC-CDMA transmit signal is obtained after processing the sequence $S$ in the OFDM block. The received signal $r$ consists of the transmitted signal plus an additive interference denoted by $n$. Hence

$\mathrm{r}=\mathrm{Hs}+\mathrm{n}$

Where $\mathrm{H}$ is the channel matrix. To recover the original message signal the received sequence given by the equation (3) is first processed in inverse OFDM block. Processed signal is despread using the Walsh- Hadamard codes which is synchronized with the transmitter code. Demodulated signals are decoded with Viterbi algorithm which performs maximum likelihood decoding of convolutional codes.

\subsection{Convolution coding}

Convolution coding is used to reduce the bit errors introduced during the transmission of a modulated signal through a wireless channel. In convolutional codes, each block of $\mathrm{k}$ bits is mapped into a block of $\mathrm{n}$ bits to be transmitted over the channel, but these $\mathrm{n}$ bits are not only determined by the present k-information bits but also by the previous information bits. This dependence on the previous information bits causes the encoder to be a finite state machine [5]. Specifically, the binary input data is shifted into each stage of the shift register $\mathrm{k}$ bits at a time, and each of these shifts produces a coded sequence of length $n$ [6]. The rate of the code is.

$$
R_{c}=\frac{k}{n}
$$

Decoding of the convolutional codes is done using Viterbi algorithm has been used which performs maximum likelihood decoding of convolutional codes [6].

\subsection{Digital Modulation Schemes}

For the transmission over analog channel data bits are mapped to the signal waveforms. This process of mapping data bits to the signal waveforms is known as digital modulation [7] [8].

\subsubsection{Binary Phase Shift Keying (BPSK)}

In BPSK, carrier signal phase is switched between two values corresponding to binary 1 and 0 , respectively. Normally, the two phases are separated by $180^{\circ}$ [7][8]. If the sinusoidal carrier has an amplitude $A_{c}$ and energy per bit $E_{b}$, then the transmitted BPSK signal for $0 \leq t \leq T_{b}$ is either: 


$$
\begin{aligned}
& S_{B P S K}(t)=\sqrt{\frac{2 E_{b}}{T_{b}}} \cos \left(2 \pi f_{c} t+\theta_{c}\right)(\text { binary } 1) \\
& S_{B P S K}(t)=-\sqrt{\frac{2 E_{b}}{T_{b}}} \cos \left(2 \pi f_{c} t+\theta_{c}\right)(\text { binary } 0) \\
& \text { Where } \quad E_{b}=\frac{1}{2} A_{c}^{2} T_{b}
\end{aligned}
$$

\subsubsection{Quadrature Phase Shift Keying (QPSK)}

QPSK is also known as four level PSK in which signal carries one bit per symbol interval on both the in-phase and quadrature-phase component. In QPSK two bits are transmitted in a single modulation symbol which doubles the bandwidth efficiency of QPSK as compare the efficiency of BPSK [8][9]. QPSK signals are defined as

$$
\begin{gathered}
s_{i}(t)=\sqrt{\frac{2 E}{T} \cos \left(2 \pi f_{c} t+\theta_{i}\right)} \\
\qquad i=1,2,3,4 \\
\theta_{i}=\frac{(2 i-1) \pi}{4}
\end{gathered}
$$

Where $\mathrm{E}$ denotes the transmitted signal energy per symbol, $\mathrm{T}$ is symbol duration and $\mathrm{fc}$ is carrier frequency. The initial signal phases are $\pi / 4,3 \pi / 4,5 \pi / 4$ and $7 \pi / 4$.

\subsection{Combining Techniques}

To combine the multiple copies of same information containing signal at the receiver end two combining techniques which are MRC and EGC has used.

\subsubsection{Maximal Ratio Combining (MRC)}

In MRC signals received from multiple the paths are must be co-phased first individually then weighted according to their individual signal voltage to noise power ratios and summed. After combining the multiple signals using MRC output SNR is equal to the sum of the individual SNRs. MRC receiver can produce an output with an acceptable SNR even in the absence of any of the individual signals with acceptable SNR [8][9].

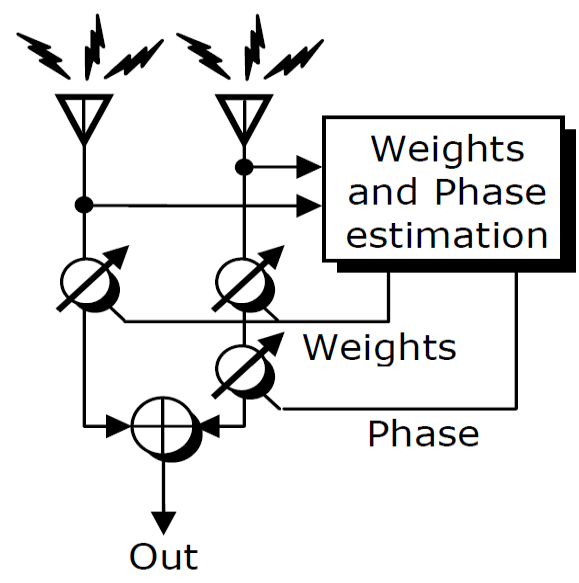

Figure 3.14 Maximum ratio combining [7]

\subsubsection{Equal Gain Combining (EGC)}

In EGC after processing the multiple copies of the same signal each replica is considered with equal weight then sum them to produce the decision statistic. In EGC receiver estimation of the channel carrier phase is required but the weights applied to each branch in the combiner are complex quantities whose amplitudes are all set to 1. EGC provide comparable performance to the MRC with less receiver complexity [8] [9].

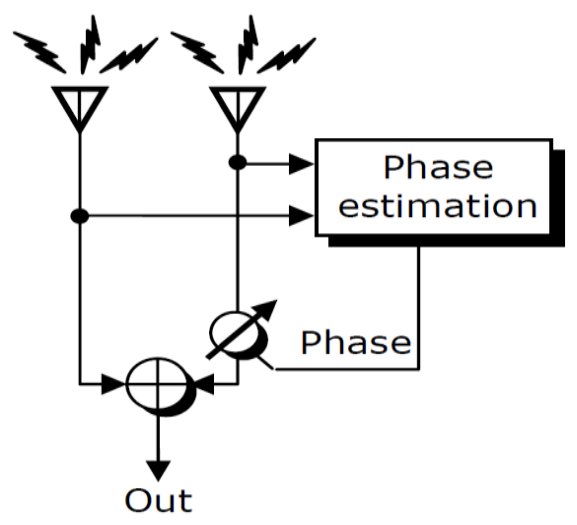

Figure 3.15 Equal gain combining [7].

\section{SIMULATION RESULTS}

In this section, we present and discuss the simulation results of the BER Vs SNR performance of $1 / 2$ rate convolution coded Multicarrier CDMA system over frequency selective Rayleigh channel with two modulation schemes (BPSK and QPSK) and two combining techniques (EGC and MRC) through MATLAB. Simulation parameter is given in table 1.

\subsection{Bit Error Rate (BER)}

BER is defined as the ratio of number of errors to total number of bits transmitted

$$
B E R=\frac{\text { Number of Errors }}{\text { Total no of Bits Transmitted }}
$$

\subsection{Signal to Noise Ratio (SNR)}

\begin{tabular}{|c|c|}
\hline Parameters & Value \\
\hline Number of input data bits & 10000 \\
\hline Number of sub-carriers & 16 \\
\hline Number of users & $2,8,16$ \\
\hline Channel coding & Convolutional coding \\
\hline Code rate & $1 / 2$ \\
\hline Spreading codes & Walsh-Hadamard codes \\
\hline Modulation techniques & BPSK,QPSK \\
\hline Channel model & Rayleigh \\
\hline Spreading code length $L$ & 16 \\
\hline FEC coding & None \\
\hline Diversity combining & MRC, EGC \\
\hline
\end{tabular}

SNR is defined as the ratio of signal power to noise power it is usually measured in decibel. The SNR mathematically can be expressed as follows:

$$
S N R_{d b}=10 \log _{10}\left(\frac{P_{\text {signal }}}{P_{\text {noise }}}\right)
$$

Table 1: Simulation Parameters 


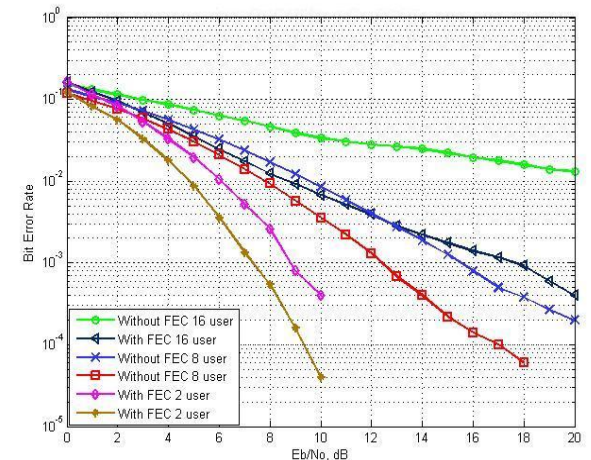

Fig 2. BER Vs SNR performance of MC-CDMA with EGC using BPSK over Rayleigh channel

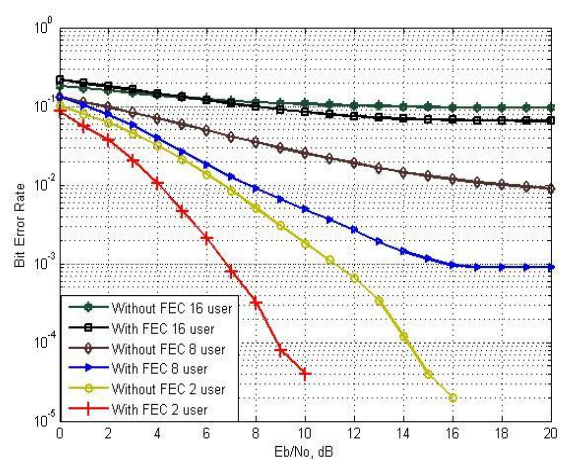

Fig 3. BER Vs SNR performance of MC-CDMA with MRC using BPSK over Rayleigh channel

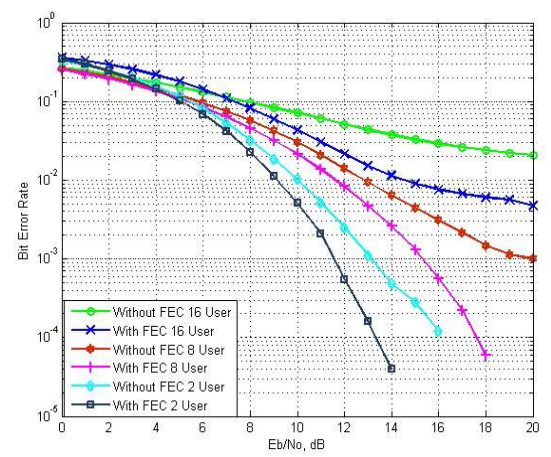

Fig 4. BER Vs SNR performance of MC-CDMA with EGC using QPSK over Rayleigh channel

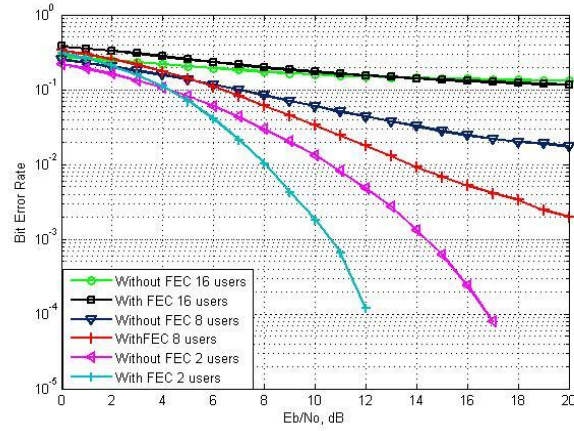

Fig 5. BER Vs SNR performance of MC-CDMA with MRC using QPSK over Rayleigh channel

\section{CONCLUSION}

Simulation results are given in section 3 and it shows that the performance of MC-CDMA system is improved by an significant amount when convolution codes are applied as FEC codes along with BPSK and QPSK modulation techniques for MRC and EGC combining techniques. Comparison is made against the BER and SNR performance of MC-CDMA system with and without FEC codes along with two modulation techniques i.e. BPSK and QPSK for different number of users. Simulation results shows that the MRC provides better SNR performance for a MC-CDMA system as compare to EGC when number of users are less but EGC outperforms the MRC when the number of users in the system are increases. Simulation results also shows that MCCDMA performs better when BPSK is used instead of QPSK. In future this work can be extended for different modulation techniques and FEC coding techniques over Rician and Nakagami channel models.

\section{REFERENCES}

[1] K. Fazel and S. Kaiser, Multi-Carrier and Spread Spectrum Systems. New York: Wiley, 2003.

[2] S. Hara and R. Prasad "Overview of multicarrier CDMA,” IEEE Commun. Mag., pp. 126-133, Dec. 1997.

[3] Antonia M. Tulino, Linbo Li, and Sergio Verdu "Spectral Efficiency of Multicarrier CDMA" IEEE Transactions on Information Theory, Vol. 51, No. 2, February 2005.

[4] S.Kuzhaloli and K.S.Shaji "Comparison of Equalization Techniques for an MIMO MC-CDMA system" IEEE International Conference on Circuit, Power and Computing Technologies, 2015

[5] John G. Proakis and Masoud Salehi "Communication Systems Engineering” Prentice-Hall, 2002

[6] Andrea Goldsmith "Wireless Communications" Cambridge University Press, 2005

[7] Andreas F. Molisch "Wireless Communications" Second Edition Wiley, 2011.

[8] Anita Mukati and Angeeta Hirwe "Performance Evaluation of MC-CDMA System with MRC and EGC Diversity Techniques over Rayleigh Fading Channel" International Journal of Engineering Applied Sciences and Technology, Vol. 1, No. 11, October 2016

[9] Theodore S. Rappaport "Wireless Communication Principal and Practice" Second Edition, Inc. Pearson ed., 2002. 